\title{
Ultrasound Technique for Prediction of Statistical Characteristics of Grid-Generated Turbulence
}

\author{
T. A. Andreeva* and W. W. Durgin ${ }^{\dagger}$ \\ Worcester Polytechnic Institute, Worcester, Massachusetts 01609
}

\begin{abstract}
Ultrasonic measurement techniques can be used to determine some characteristics of turbulent flows. In this work measurements of the difference in propagation times of ultrasonic waves along two paths are combined with the Kolmogorov $\left(\frac{2}{3}\right)$-power law to study characteristics of grid-generated turbulence. The paper focuses on ultrasonic measurements of turbulent flow using the travel-time technique. The present research is of interest from two points of view. First, it demonstrates an ultrasonic method of investigation of turbulence, and second, it furthers understanding of the effect of turbulence on sound propagation.
\end{abstract}

\section{Introduction}

$\mathbf{T}$ ECHNICAL advances of ultrasonic flow metering have drastically improved the accuracy of flow measurements. A thorough description of ultrasonic metering can be found in Lynnworth.' The technological capabilities developed for the ultrasonic technique flow measurement, namely, measurement of very small time differences associated with changes in the ultrasound wave propagation, can be utilized beneficially for turbulent flow diagnostics.

The influence of turbulence on sound wave propagation has been studied by a number of authors, who conducted a variety of experiments and provided a wide range of experimental and analytical results in this area for the past 50 years. However, the experimental results were not systematically acquired or presented. Furthermore, early results were not accurate because of poor experimental setups and technological limitations of timing equipment. For instance, in the work by Krasilnokov, ${ }^{2}$ which will be discussed later, the measurements were conducted outdoors and assumed that the atmospheric boundary-layer turbulence was isotropic. Strictly speaking, this assumption could not be satisfied during the experiments, which resulted in questionable accuracy of the results. There continues to be a strong interest in understanding of the propagation of sound in an inhomogeneous, moving medium. An analytical relationship between phase fluctuations in sound and atmospheric turbulence was first formulated by Krasilnikov ${ }^{2}$ Bovsheverov and Krasilnikov ${ }^{3}$ and developed further by Chernov. ${ }^{4}$ The authors studied the timestructural phase functions at the points of reception in the sonic and ultrasonic range for various distances from a speaker to a microphone and various frequencies of sound as well as the dependence of the amplitude pulsation intensity on a distance. Substantial work was performed by Suchkov, ${ }^{5}$ who investigated the amplitude fluctuations of sound at sonic and ultrasonic frequencies. Similar work was carried out by Herbstreit and Thompson ${ }^{6}$ and Deam and Fannin, where measurements of phase difference fluctuations of microwaves were made. Although the literature is replete with results of measurements of sound propagation through the atmosphere, very few data are available that include the detailed properties of the atmosphere at the time of measurements. An attempt to develop comprehensive theory along with experiment was made by Wiener and Keast. ${ }^{8} \mathrm{~A}$

Received 26 November 2001; presented as Paper 2002-0921 at the 40th Aerospace Sciences Meeting, Reno, NV, 14-17 January 2002; revision received 11 February 2003; accepted for publication 16 February 2003. Copyright (C) 2003 by the American Institute of Aeronautics and Astronautics, Inc. All rights reserved. Copies of this paper may be made for personal or internal use, on condition that the copier pay the $\$ 10.00$ per-copy fee to the Copyright Clearance Center, Inc., 222 Rosewood Drive, Danvers, MA 01923; include the code 0001-1452/03\$10.00 in correspondence with the CCC.

${ }^{*}$ Graduate Student, Mechanical Engineering Department, 100 Institute Road; tatiana@wpi.edu.

${ }^{\dagger}$ Professor, Mechanical Engineering Department, 100 Institute Road; wwdurgin@wpi.edu. Associate Member AIAA. very comprehensive review of theoretical and experimental work done in the area of wave propagation in random media can be found in Tatarskii, ${ }^{9}$ Rytov et al., ${ }^{10}$ and Ishimaru. ${ }^{11}$ More recently, the problem of pulse propagation in inhomogeneous and random media became of interest in applied science and has been investigated using different approaches by different researches (Ref. 12 and references therein). Although a large number of atmospheric measurements were made, they suffered a lack of reliability and accuracy in addition to poor characterization of the turbulence. The problem of phase fluctuations is equivalent to the problem of finding the autocorrelation functions of these fluctuations, which involves enormous amounts of experimental data and a large amount of computational work. From the repeatability of experiments point of view, it is much more complicated and time-consuming to conduct outdoor experiments compared to those performed under convenient laboratory conditions. The break through in the problem of measuring flow parameters in a turbulent medium was made by Schmidt and Tilmann ${ }^{13}$ and Schmidt ${ }^{14}$ who discovered the possibility of measuring flow parameters noninvasively and without perturbation by means of ultrasound. Johari and Durgin ${ }^{15}$ reported a number of applications that extended Schmidt's initial work. Specifically, they investigated unsteady flow about an airfoil, the trailing vortex from a delta wing, and swirling free-surface flows.

During the past 25 years, ultrasonic technology has progressed very rapidly in improving flow measurement performance and resulted in the development of the equipment capable of measuring the very small time differences associated with changes in the ultrasound wave propagation time. A description of ultrasonic metering can be found in Lynnworth. ${ }^{1}$ The study of the transmission and attenuation of the signal and noise mechanism performed by Brassier et al. ${ }^{16}$ gives us a defined choice of the frequency at which ultrasonic transducers can be operated. In their work authors presented innovated prototype of the ultrasonic flow meter using optimal choice of ultrasonic frequency, the design, and the "echo process." With improved technology we are now able to measure volume flow rate and other flow parameters in pipes and conduits reliably and accurately in laboratory-scale apparatus.

A theoretical basis of the present work is based on well-known Kolmogorov law, derived purely from the dimensional analysis. Namely, it states that the velocity fluctuations at two different points are proportional to the distance between these points to the power $\frac{2}{3}$, and therefore sometimes it is called " $\frac{2}{3}$ law." $17-19$

Our interest is concentrated on the effect of turbulence on sound wave propagation. The random changes of flow parameters produced by turbulence are very rapid and affect the sound propagation. This area of research lies on the boundary between acoustics and aerodynamics.

The research has a two-fold purpose: first is the application of travel-time ultrasonic techniques for data acquisition in the gridgenerated turbulence produced in a wind tunnel, and the second is to compare an analytical expression for phase fluctuations based on 
Kolmogorov's $\frac{2}{3}$ law, with experimental data to validate experimental setup. Thus, the paper will present the results of two series of experiments on the statistics of time propagation as a function of distance and propagation velocity.

The plan of this paper is as follows. The methodology is presented in Sec. II. The experimental setup is described in Sec. III. The experimental results and comparisons are discussed in Sec. IV, and in Sec. V conclusions will be presented.

\section{Methodology}

In the experimental part of the investigation, the authors use ultrasonic pulses traveling along straight paths from a single source to the receiver, as shown in Fig. 1. For the work reported herein, the sound propagates a distance $L$ across the grid-generated turbulence from a speaker to microphone. The flow-meter equation can be used to derive an expression for a travel time of a wave traveling from the speaker to icrophone $t_{1}$ and in opposite direction $t_{2}$. These travel times can be written as

$$
\begin{aligned}
& t_{1}=\int_{0}^{L} \frac{\mathrm{d} y}{c-u_{1}} \approx t_{0}+\frac{1}{c^{2}} \int_{0}^{L} u_{1} \mathrm{~d} y, \quad u_{1}=U \sin \beta+u_{1}^{\prime} \\
& t_{2}=\int_{0}^{L} \frac{\mathrm{d} y}{c+u_{2}} \approx t_{0}-\frac{1}{c^{2}} \int_{0}^{L} u_{2} \mathrm{~d} y, \quad u_{2}=U \sin \beta+u_{2}^{\prime}
\end{aligned}
$$

where $t_{0}$ is a travel time in the undisturbed media, $U$ is a mean velocity, $c$ is a sound speed, and $u^{\prime}$ are fluctuations of the mean flow velocity. In Eq. (1) we neglected the terms of order $U / c, U^{2} / c^{2}$. Then, for the time difference we find

$$
\Delta t=t_{2}+t_{1}-2 t_{0} \approx \frac{1}{c^{2}} \int_{0}^{L}\left(u_{1}^{\prime}-u_{2}^{\prime}\right) \mathrm{d} y=\frac{1}{c^{2}} \int_{0}^{L} \Delta u \mathrm{~d} y
$$

However, the turbulent velocity fluctuations on which travel time of the wave depends appear as a random function of time and position. Then, from Eq. (2)

$$
\overline{\Delta t^{2}}=\frac{1}{c^{4}} \int_{0}^{L} \mathrm{~d} y^{\prime} \int_{0}^{L} \mathrm{~d} y^{\prime \prime} \overline{\Delta u\left(y^{\prime}\right) \Delta u\left(y^{\prime \prime}\right)}
$$

where the overscore indicates time averaging. The principal change in the total velocity $u$ is caused by the drift of the turbulence in the mean flow, so that the change in the velocity $u$ in the time $t$ cin be represented as the result of a displacement of the turbulence in a small distance $\delta(\vartheta)=u t$ as shown in Fig. 2 for the case of perpendicular direction of the sound wave. The underintegral term in Eq. (3) is

$$
\begin{gathered}
\overline{\Delta u\left(y^{\prime}\right) \Delta u\left(y^{\prime \prime}\right)}=\overline{\left[u\left(y^{\prime}, 0\right)-u\left(y^{\prime}, \delta\right)\right]\left[u\left(y^{\prime \prime}, 0\right)-u\left(y^{\prime \prime}, \delta\right)\right]} \\
=\overline{u\left(y^{\prime}, 0\right) u\left(y^{\prime \prime}, 0\right)}+\overline{u\left(y^{\prime}, \delta\right) u\left(y^{\prime \prime}, \delta\right)} \\
-\overline{u\left(y^{\prime}, \delta\right) u\left(y^{\prime \prime}, 0\right)}-\overline{u\left(y^{\prime}, 0\right) u\left(y^{\prime \prime}, \delta\right)}
\end{gathered}
$$

To take into account the correlation of fluctuations at different points of the flow, we use the $\frac{2}{3}$ law obtained independently by A. N. Kolmogorov ${ }^{17.18}$ and A. M. Obukhov. ${ }^{19}$ Following this law, one may get

$$
\overline{\left[u\left(y^{\prime}\right)-u\left(y^{\prime \prime}\right)\right]^{2}}=C^{2} R^{3}
$$

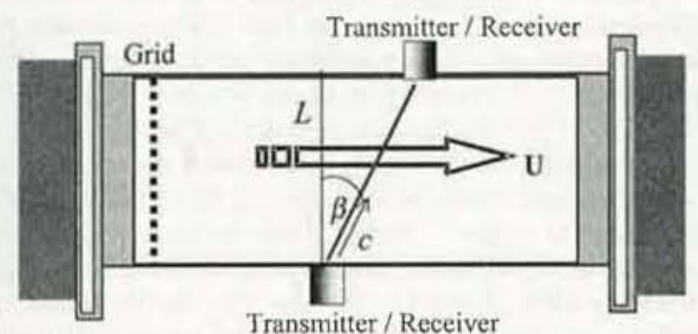

Fig. 1 Sketch of the wind-tunnel test section with ultrasonic flow meter.

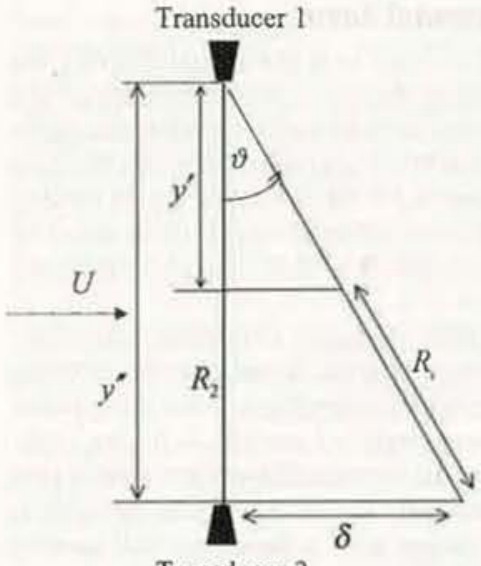

Transducer 2
Fig. 2 Sketch for the basic relations for the ultrasound measurement. where constant $C$ is the characteristic of turbulence having a dimension of $1 \mathrm{~cm}^{2 / 3} \times \mathrm{s}^{-1}$ and $R$ is a distance between the points $y^{\prime}$ and $y^{\prime \prime}$. However, based on the hypotheses of isotropy of the turbulence the left-hand side of Eq. (5) can be written as follows:

$$
C^{2} R^{2}=\left[u\left(y^{\prime \prime}\right)-u\left(y^{\prime \prime}\right)\right]^{2}=2\left\{\left[u\left(y^{\prime}\right)\right]^{2}-u\left(y^{\prime}\right) u\left(y^{\prime \prime}\right)\right\}
$$

Based on the Fig. 2, it is seen that

$$
\begin{aligned}
& R_{1}=R\left[\left(y^{\prime}, \delta\right),\left(y^{\prime \prime}, 0\right)\right]=R\left[\left(y^{\prime}, 0\right),\left(y^{\prime \prime}, \delta\right)\right] \\
& R_{2}=R\left[\left(y^{\prime}, 0\right),\left(y^{\prime \prime}, 0\right)\right]=R\left[\left(y^{\prime}, \delta\right),\left(y^{\prime \prime}, \delta\right)\right]
\end{aligned}
$$

Simple geometry for small $\vartheta$ gives the following:

$$
\begin{gathered}
R_{1}^{2}=\delta^{2}+\left(y^{\prime}-y^{\prime \prime}\right)^{2} \\
R_{2}^{2}=\left(y^{\prime \prime}-y^{\prime}\right)^{2}
\end{gathered}
$$

Now making use of the $\frac{2}{3}$ law and Eqs. (9) and (10), one obtains

$$
\begin{aligned}
& \overline{\Delta u\left(y^{\prime}\right) \Delta u\left(y^{\prime \prime}\right)}=C^{2}\left\{R_{1}^{\frac{2}{3}}-R_{2}^{\frac{2}{3}}\right\} \\
& =-0.5 C^{2}\left\{2\left(y^{\prime}-y^{\prime \prime}\right)^{\frac{2}{3}}-2\left[\left(y^{\prime}-y^{\prime \prime}\right)^{2}+\delta^{2}\right]^{\frac{1}{3}}\right\}
\end{aligned}
$$

Substitution of Eq. (11) into Eq. (3) and integration yields the following result:

$$
\overline{\Delta t^{2}}=C^{2} L\left(1 / c^{2}\right)^{2} \delta^{\frac{5}{5}} \text { const }
$$

in which the const was determined experimentally in the works by Obukhov ${ }^{19.20}$ to be equal to three. Then, standard deviation is the square of distance, agreeing with results of other authors ${ }^{2.19 .20}$ :

$$
\sigma=\sqrt{\overline{\Delta t^{2}}}=\sqrt{3} C L^{\frac{1}{2}}\left(1 / c^{2}\right)(\delta)^{\frac{5}{6}}
$$

The experiment falls into two parts. First, to validate our experimental equipment we use the data to compare theoretically derived dependence of the standard deviation of the travel time on the separation distance $L$. Here we collect data of transit time at constant mean velocity for nine different distances. Second, we attempt to estimate the standard deviation of the travel time and verify its dependence on a mean flow velocity. For this purpose we conduct the second series of experiments, where we collect data for six different mean flow velocities. Finally, after validating our experimental equipment and data we estimate the structure parameter $C$ based on experimental data and perform the comparison with experimental results of research activities carried out in earlier years. 


\section{Experimental Arrangement}

The experiments were carried out in a wind tunnel of $1.15-\mathrm{m}$ length with a $0.3 \times 0.3 \mathrm{~m}$ rectangular test section. Turbulence was produced by a biplanar grid consisting of a square mesh of aluminum round rods with diameter of $0.635 \mathrm{~cm}$ positioned $2.54 \mathrm{~cm}$ between centers. The mesh $M$ was therefore $2.54 \mathrm{~cm}$, and the grid solidity was 0.64 . The domain for the data collection was chosen based on criteria for achievement of isotropic flow developed by Mohamed and LaRue. ${ }^{21}$

In our experiment we have used ultrasonic transducers with a frequency of $100 \mathrm{kHz}$ designed for air applications. Ultrasonic bursts were generated using a programmable signal generator and a power amplifier. Received signals were amplified and sampled using highspeed data-acquisition equipment. A transmit/receive switch protected the receiver from transmitted signal. The enabling pulse is started by the first positive voltage step in the four-pulse burst of square waves. The burst was $100-\mathrm{kHz}$ frequency and had $50-\mathrm{mV}$ amplitude produced by function generator, which, in turn, was driven by a National Instrument Data Acquisition Card (DAQ). The fourpulse burst was sent to the transducer-transmitter through the amplifier. Figure 3 demonstrates four-pulse burst coming out of the transducer-transmitter.

The National Instrument DAQ allows one to detect the burst departure time extremely accurately. The pulse was generated with a repetition frequency of $500 \mathrm{cycles} / \mathrm{s}$. The ultrasound beam, sent by the first transducer, was received by the second one. The main problem was to measure travel time $t_{n}$ with a high precision. The analog data, then, from the second transducer were transported to a CompuScope 82G DAQ with large acquisition memory and wide analog bandwidth, which transformed analog data to digital data and transferred data from CompuScope $82 \mathrm{G}$ card to the $\mathrm{PC}$ memory with the

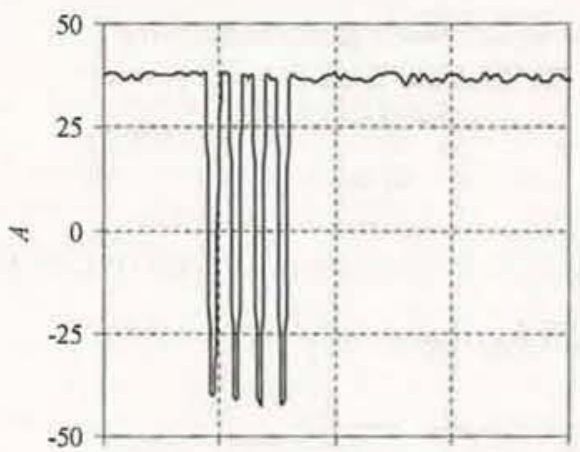

1.95E-03 2.00E-03 2.05E-03 2.10E-03 2.15E-03

$t$ (sec)

Fig. 3 Four-pulse burst of square shape waves.

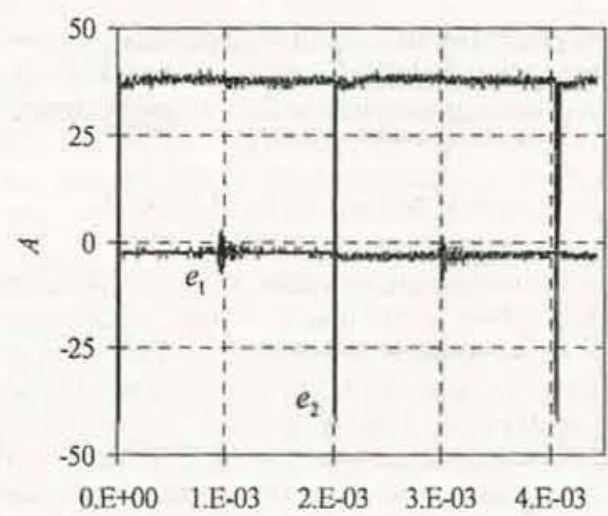

$t$ (s)

Fig. 4 Typical data representation obtained from CompuScope 82G DAQ, transferred to the PC and processed in Excel. Signals $e_{1}$ and $e_{2}$ are received and transmitted signals, respectively.

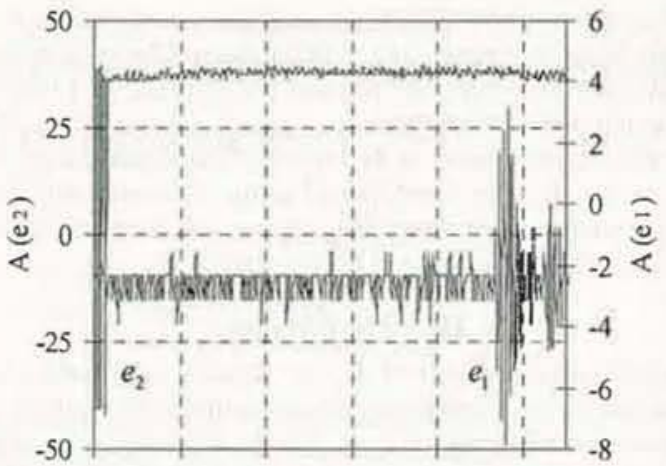

0.E+00 2.E-04 4.E-04 6.E-04 8.E-04 1.E-03

$t(\mathrm{~s})$

Fig. 5 Magnification of received signal $e_{1}$ and transmitted signal $c_{2}$, obtained from the digital data-acquisition system CompuScope 82 .

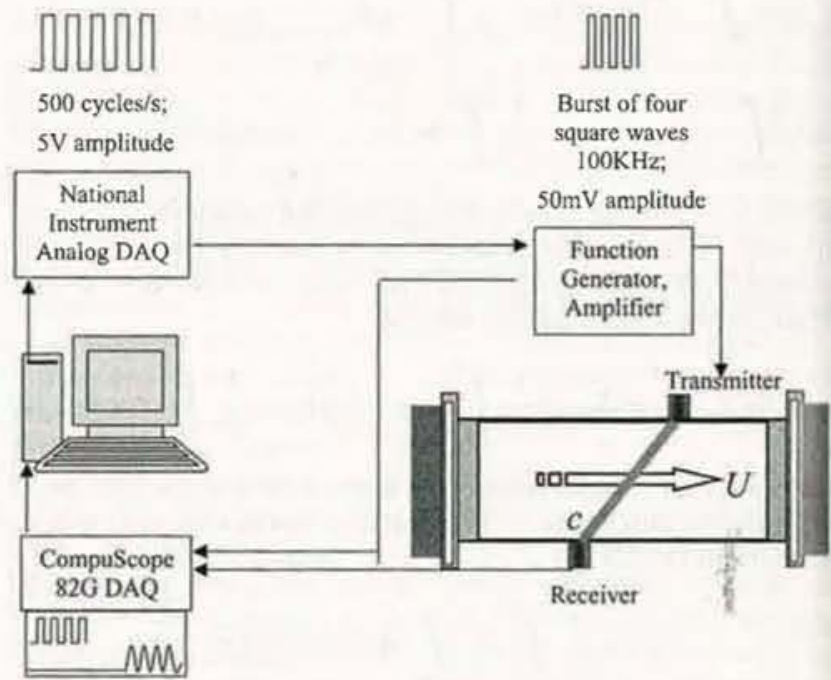

Fig. 6 Block diagram of analog and digital processing.

resolution of $5 \times 10^{-9} \mathrm{~s}$. Both DAQ cards were installed inside the PC. Digital representation of the experimental data, provided by the DAQs, allowed determination of the travel time $t_{n}$ very precisely, Figure 4 demonstrates a typical data representation obtained from CompuScope 82G DAQ, transferred to the PC, and processed in Excel. The acquisition rate was $50,000,000$ samples/s. Two signals shown in Fig. 4 are received signal $e_{1}$ and transmitted signal $e_{2}$. For this case the uitrasonic signal traversed the flow perpendicular to the mean flow. Approximately $0.9 \mathrm{~ms}$ was required. A magnification of the transmitted and received bursts is shown in Fig. 5. The block diagram of analog and digital processing is shown in Fig. 6.

\section{Experimental Results and Discussion}

We consider here a locally isotropic turbulent flow under constant room temperature, which can be approximately generated by introducing a grid into a uniform flow. At a sufficient distance down. stream from the grid, the flow becomes locally isotropic, and a power law is applicable. Studies and criteria that relate to identification of the downstream position, where the flow becomes nearly homogeneous, isotropic, and locally isotropic, are described in detail by Grant and Nisbit,, ${ }^{22}$ Corrsin, ${ }^{23}$ Uberoi and Wallis, ${ }^{24.25}$ and Mohamed and LaRue. ${ }^{21}$ To collect experimental data in nearly isotropic and homogeneous portions of flow, the locations of transducers as well as mean flow speed were chosen based on the criteria established by Mohamed and LaRue. ${ }^{21}$ In the present study we assume that the basic characteristics of ultrasound propagation in the turbulence are determined by slow changes in the state of medium (mean speed, temperature, and density of the air). Under these conditions it is expedient to use the method of ray acoustics. Furthermore, it seems

reaso

bulen

and $F$

The

averz

and

were

$15 \mathrm{~N}$

from

whe

tion

Het

Thi

vol

val

for

by

nct

III

A.

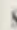

P

w

,

,



, 
reasonable that ultrasonic pulses have negligible effect on the turbulence because they are at very high frequency, are at low power, and propagate in very short bursts.

Each transducer acted both as a transmitter and as a receiver. The differences in transit times along the path were measured and averaged over more than 700 realizations. For each mean velocity and separation distance the experiment was carried out, and data were collected for $45 \mathrm{~s}$. The data file for each measurement was about $15 \mathrm{Mb}$. The transit time for the ultrasound pulse was determined from the correlation function

$$
K_{12}(\tau)=\overline{e_{1}(t+\tau) e_{2}(t)}
$$

where $K_{12}(t)$ is a cross-correlation function of $e_{1}$ and $e_{2}$. By definition

$$
K_{12}(\tau)=\frac{1}{T} \int_{0}^{T} e_{1}(t+\tau) e_{2}(t) \mathrm{d} t
$$

Hence, the travel time $t_{n}$ can be determined as

$$
\max K_{12}(\tau)=K_{12}\left(t_{n}\right)
$$

The numerical computation of the cross-correlation function involves the computation of average products among the sample data values. ${ }^{26}$ In the following two subsections we describe experiments for two different setups performed to verify our experimental data by comparing it with theoretical estimations. Next, turbulent characteristic parameter is obtained on the basis of Kolmogorov's theory and compared to experimental data.

\section{A. Transit Time as a Function of the Separation Distance $L$}

Figure 7 represents the schematic diagram for the experimental setup. Nine cases of different distances $L$ were studied. Geometrical parameters are listed in the Table 1, and it can be seen that the angle with respect to the mean flow varied so that the mean flow component along the path also varied. The mean velocity during the experiment was $U=3.5 \mathrm{~m} / \mathrm{s}$, which corresponded to a Reynolds number based on the mesh size of $R e \cong 7 \times 10^{3}$. The signal was sent in a direction opposite to the inlet flow. From theoretical point of view, one can expect that the travel time will increase with distance. In Fig. 8 the travel-time data in disturbed medium are plotted along with data collected in undisturbed medium and compared with theoretical estimation of the travel time without taking into account the effect of turbulence, namely, $t_{\text {theoreticat }}=L /(c-U \sin \beta)$. Figure 8 demonstrates the well-expected result of significant decrease of mean travel

\section{Table 1 Geometrical parameters}

\begin{tabular}{ll}
\hline \hline$\beta$, deg & \multicolumn{1}{c}{$L, \mathrm{~m}$} \\
\hline 0 & 0.33 \\
5 & 0.333448 \\
10 & 0.339499 \\
15 & 0.348339 \\
20 & 0.360278 \\
25 & 0.375772 \\
30 & 0.395484 \\
35 & 0.420361 \\
40 & 0.451762 \\
\hline
\end{tabular}

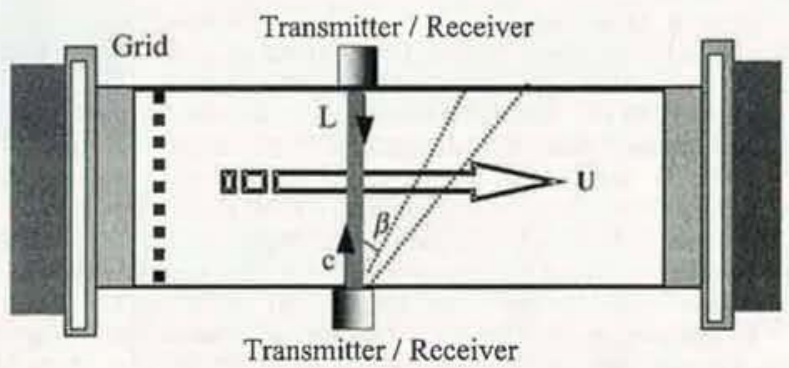

Fig. 7 Sketch of wind-tunnel test section. time in the presence of turbulence. Figure 9 demonstrates the traveltime standard deviation as a function of traveled distance. The result obtained by Obukhov ${ }^{19,20}$ predicts standard deviation increase proportional to the square root of a distance, which is depicted by the solid line. Therefore, the experimental data are plotted together with $L^{1 / 2}$ curve in order to verify that the standard deviation obtained experimentally indeed is proportional to $L^{1 / 2}$. Although the standard deviation is increasing with distance, the scattering of the data can be clearly seen. The scattered data must be interpreted with some caution and cannot be inconsistent with the theoretical analysis, but rather might be caused by the uncertainty of the measurements, which were found to be around $20 \%$.

\section{B. Transit Time as a Function of Mean Velocity}

For this case we have two transducers placed at a distance $L$ from each other, and the direction of mean flow is perpendicular

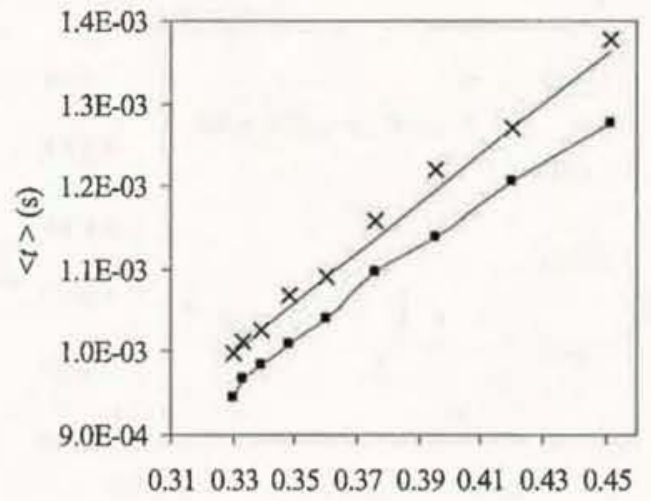

$L(\mathrm{~m})$

Fig. 8 Average travel time vs a path length: $a$, experimental data with turbulence; - theoretical estimate without turbulence; and $x$, experimental data without turbulence.

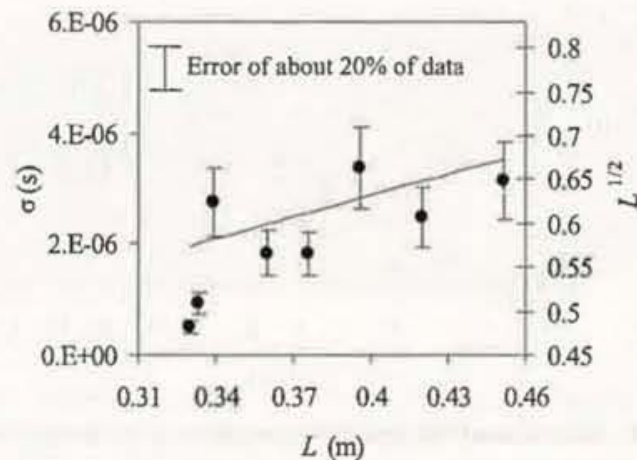

Fig. 9 Standard deviation of the travel time vs a distance: •, experiment, left axis and - $\longrightarrow$, theory, right axis.

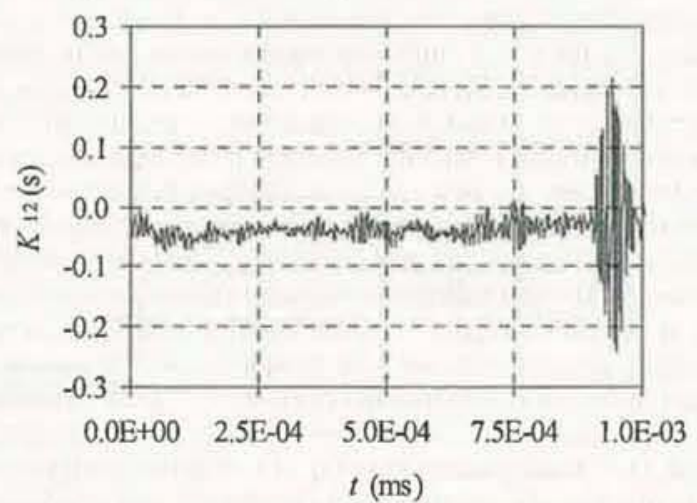

Fig. 10 Cross-correlation function $K_{12}(t)$ of two waves $e_{1}, e_{2}$. 


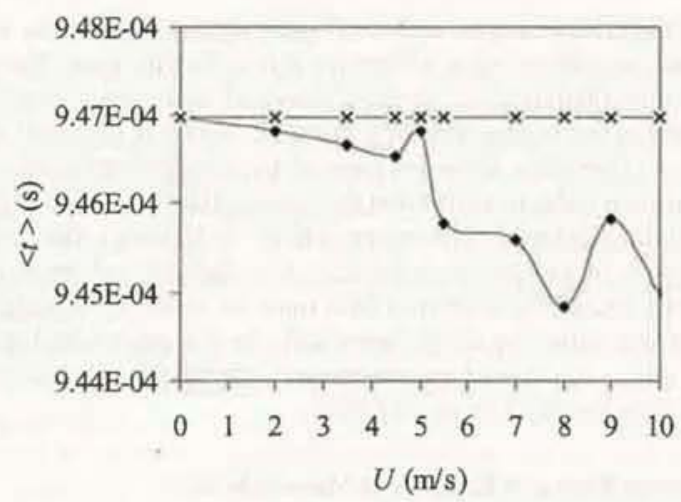

Fig. 11 Averaged travel time as a function of the mean velocity: $\bullet$, averaged travel time with turbulence and $\times$, averaged travel time without turbulence.

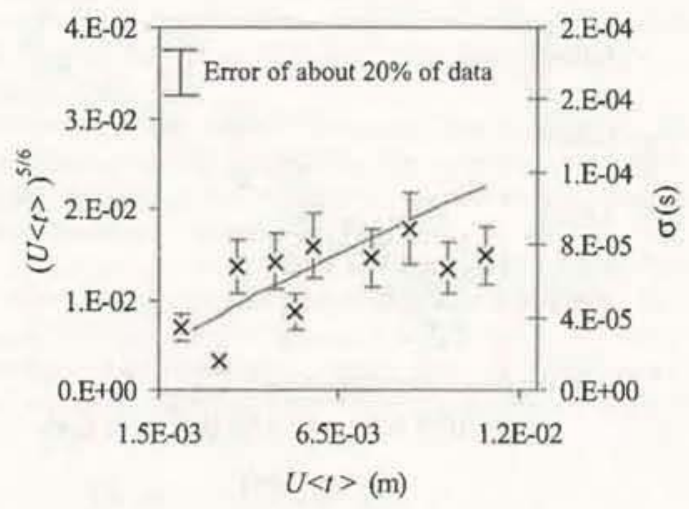

Fig. 12 Standard deviation of the travel time $\sigma$ vs mean velocity: theory, left axis and $x$, experiment, right axis.

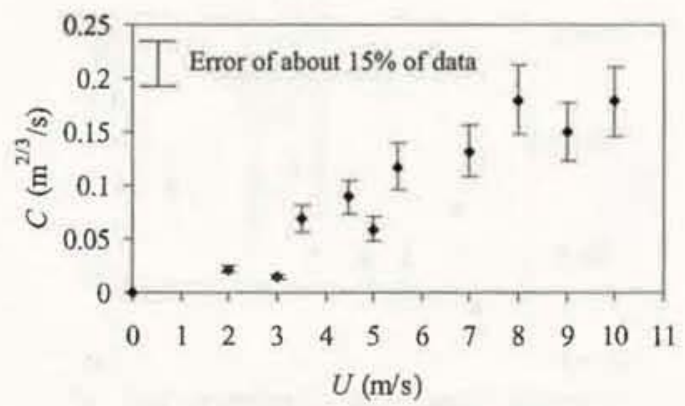

Fig. 13 Variation of the structure parameter $C$ vs mean velocity.

to $L$. The Reynolds number based on the mesh size $M$ varies from $4.016 \times 10^{3}$ to $2.0080 \times 10^{4}$. Following the procedure described in the preceding section, the cross-correlation function $K_{12}$ of two signals $e_{1}, e_{2}$ for $U=4(\mathrm{~m} / \mathrm{s})$ has been obtained and is shown in Fig. 10. After calculation of the travel time $t$ for each sample, using the formula we calculated the averaged time $\langle t\rangle$ and deviation of the transit time $\sigma$ for each velocity. Averaged travel time and deviation of the travel time are two crucial parameters that carry the most information about experimental data. As expected, Fig. 11 shows substantial decrease in $\langle t\rangle$ as mean velocity increases. As it was stated in Sec. II, for a standard deviation $\sigma$ theory predicts a dependence of $(U\langle t\rangle)^{5 / 6}$. Figure 12 illustrates that experimental results are in fairly good accordance with theoretical predictions, namely, standard deviation is proportional to $(U\langle t\rangle)^{5 / 6}$. After validation of our experimental results, we can determine the turbulent constant $C$ from Eq. (13). In accordance with Eq. (13) with the quantities entering into this formula obtained from experiment, one can determine the value of turbulent characteristic $C$. Figure 13 shows the variation of the structure parameter $C$ as a function of a velocity. Evidently. the constant $C$ increases with mean flow. This result is consistent with results obtained earlier by Krasilnikov and Obukhov. 2,19,20

\section{Conclusions}

More accurate and more systematic data regarding ultrasonic wave propagation through isotropic turbulence have been made in this work. Simple methodology using ultrasonic techniques together with Kolmogorov's $\left(\frac{2}{3}\right)$-power law has been described for diagnostics of grid-generated turbulent flow. Several experiments have been performed in a wind tunnel, and experimental data have been collected. The work has shown that an ultrasonic method can be efficiently utilized for measuring turbulent flow in laboratory scale.

In the experiment with different values of mean velocity, the experimental results have indicated that there is strong dependence on the former. Intuitively, it seems as if the mean velocity should not influence the travel time, being perpendicular to the ultrasonic signal propagation path. However, such a conclusion is wrong, and the derived analytical expression has found its justification in the numerical experiments.

Good results have been obtained from an experiment for different distances, where the influence of turbulence is clearly present. Despite data scattering, the experimental results in this case are proportional to the square of distance, agreeing with results of other authors. 2,19,20 The data scattering can be explained by two factors: the accuracy of the travel-time determination and the uncertainty of other measured parameters. Nevertheless, the behaviors are shown to be in agreement between theory and experiment.

\section{References}

'Lynnworth, L. C., Ultrasonic Measurements for Process Control, Academic Press, San Diego, CA, 1989.

${ }^{2}$ Krasilnikov, V. A., "On the Influence of the Fluctuations of the Index of Refraction in the Atmosphere on Ultrasound Wave Propagation," Izvestiya Akademii Nauk SSSR. Seriya Geograficheskaya i Geofizicheskaya, Vol. 13. No. 1, 1949, pp. 33-57.

${ }^{3}$ Bovsheverov, V. M., and Krasilnikov, V. A., "Preliminary Tosts on the Acoustic Probing of the Atmosphere with a Monochromatic Beam," Doklady Akademii Nauk SSSR, Vol. 32, 1941, pp. 44-46.

${ }^{4}$ Chernov, L. A., Wave Propagation in a Random Medium, McGraw-Hill, New York, 1960.

${ }^{5}$ Suchkov, B. A., "Amplitude Fluctuations in Sound in a Turbulent Medium," Soviet Physics-Acoustics, Vol. 4, 1958, p. 84.

${ }^{6}$ Herbstreit, J. W., and Thompson, M. C., "Measurements of the Phase of Radio Waves Received over Transmission Paths with Electrical Lengths Varying as a Result of Atmospheric Turbulence," Proceedings of the IRE, Vol. 43, 1955, pp. 1391-1401.

'Deam, A. P., and Fannin, B. M., "Phase-Difference Variations in 9350 Megacycle Radio Signals Arriving at Spaced Antennas," Proceedings of the IRE, Vol. 43, 1955, pp. 1402-1404.

${ }^{8}$ Wiener, F. M., and Keast, D. N., "Experimental Study of the Propagation of Sound over Ground," Journal of the Acoustical Society of America, Vol. 31 , No. 6,1959 , pp. $724-733$.

${ }^{9}$ Tatarskii, V. I., "The Effect of the Turbulent Atmosphere on Waves Propagation," Israel Program for Scientific Translation, Jerusalem, 1971.

${ }^{10}$ Rytov, S. M., Kravcov, Yu. A., and Tatarskii, V. 1., Introduction to Statistical Radio Physics, Random Fields, Science, Moscow, 1978.

"Ishimaru, A., Wave Propagation and Scattering in Random Media, Academic Press, New York, 1978.

${ }^{12}$ Dacol, D. K., "Pulse Propagation in Randomly Fluctuating Media," Journal of the Acoustical Society of America, Vol. 109, No. 6, 2001, pp. $2581-2586$.

${ }^{13}$ Schmidt, D. W., and Tilmann, P. M., "Experimental Study of SoundWave Phase Fluctuations Caused by Turbulent Wakes," Journal of the Acoustical Society of America, Vol. 47, 1970, pp. 1310-1324.

${ }^{14}$ Schmidt, D. W., "Acoustical Method for Fast Detection and Measurement of Vortices in Wind Tunnels," ICIASF'75 Record, pp. 216-228.

${ }^{15}$ Johari, H., and Durgin, W. W., "Direct Measurements of Circulation Using Ultrasound," Experiments in Fluids, Vol. 25, 1998, pp. 445-454.

${ }^{16}$ Brassier, P., Hosten, B., and Vulovic, F., "High-Frequency Transducers and Correlation Method to Enchance Ultrasonic Gas Flow Metering," Flow Measurement and Instrumentation, Vol. 12, 2001, pp. 201-211.

${ }^{17}$ Kolmogorov, A. N., "The Local Structure of Turbulence in Incompressible Viscous Fluid for Very Large Reynolds Numbers," C. R. Acad Sci. U.R.S.S., Vol. 30, 1941, pp. 299-303. thit

Re

Fit

st

$\mathrm{g}$ 
${ }^{18}$ Kolmogorov, A. N., "A Refinement of Previous Hypothesis Concerning the Local Structure of Turbulence in a Viscous Incompressible Fluid at High Reynolds Number," Journal of Fluid Mechanics, Vol. 13, 1961, pp. 82-85.

${ }^{19}$ Obukhov, A. M.. "Energy Distribution in the Spectrum of Turbulent Flow," Izvestiya Akademii, Nauk SSSR, Seriya Geograficheskaya i Geofizicheskaya, Vol. 5, 1941, pp. 453-463.

${ }^{20}$ Obukhov, A. M., "Some Specific Features of Atmospheric Turbulence," Journal of Fluid Mechanics, Vol. 13, 1961, pp. 77-81.

${ }^{2 !}$ Mohamed, M. S., and LaRue, J. C., "The Decay Power Law in Grid-Generated Turbulence," Journal of Fluid Mechanics, Vol. 219, 1990, pp. 195-214.

${ }^{22}$ Grant, H. L., and Nisbet, I. C.," The Inhomogeneity of Grid Turbulence,"
Journal of Fluid Mechanics, Vol. 2, 1957, pp. 263-272.

${ }^{23}$ Corrsin, S., Encyclopedia of Physics, Vol. 8, Pt. 2, 1963, p. 568.

${ }^{24}$ Uberoi, M. S., and Wallis, S., "Effect of Grid Geometry on Turbulence Decay," Physics of Fluids, Vol. 10, 1967, pp. 1216-1230.

${ }^{25}$ Uberoi, M. S., and Wallis, S., "Spectra of Grid Turbulence," Physics of Fluids, Vol. 12, 1969, pp. 1355-1358.

${ }^{26}$ Bendat, J. S., and Piersol, A. G., Random Data; Analysis and Measurement Procedures, Wiley-Interscience, New York, 1971.

R. M. C. So Associate Editor

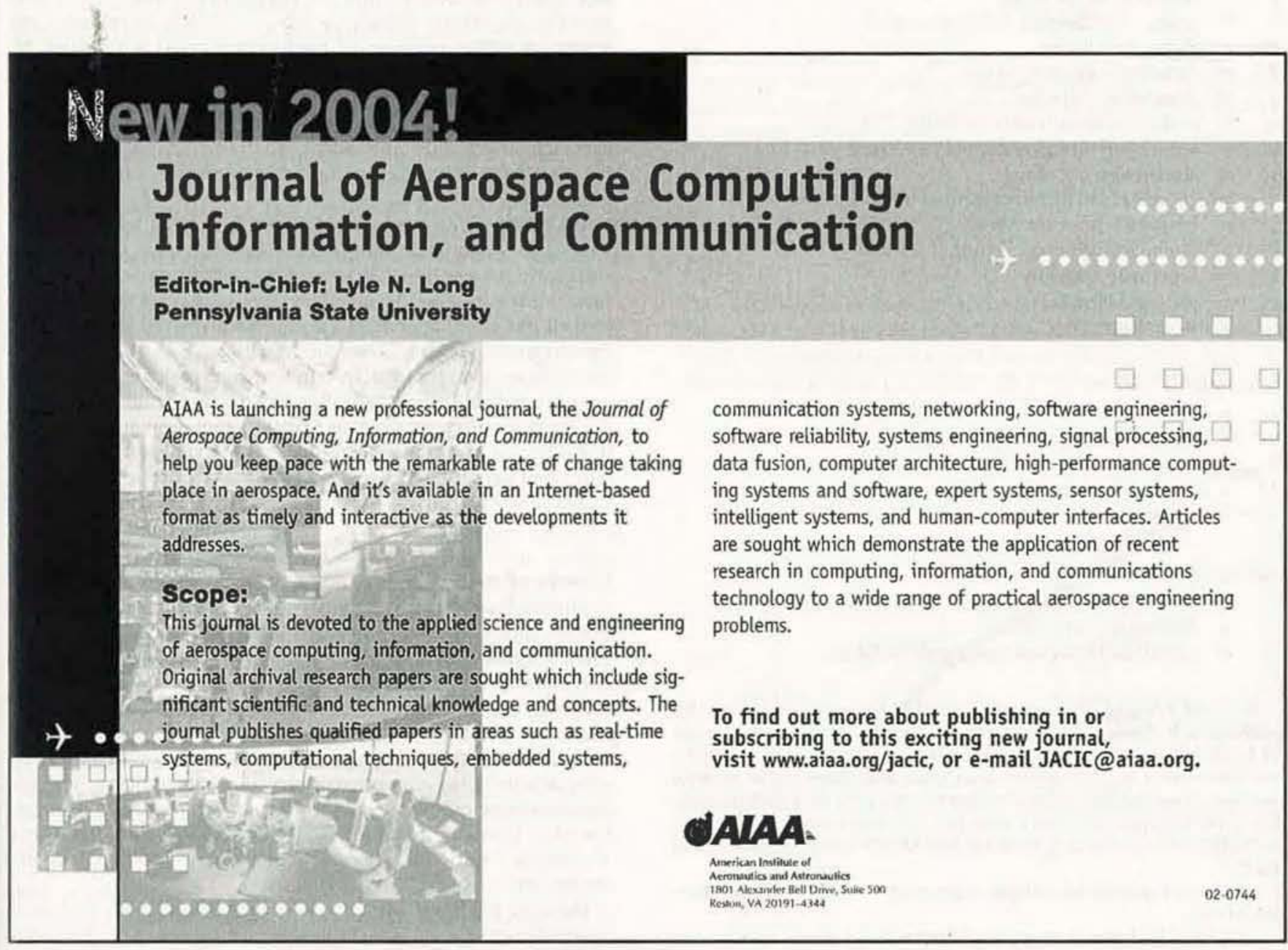

